\title{
Univariate Genetic Analysis of Oxygen Transport Regulation in Children: The Medical College of Virginia Twin Study
}

\author{
WILLIAM B. MOSKOWITZ, MICHAEL MOSTELLER, JOHN K. HEWITT, LINDON J. EAVES, \\ WALTER E. NANCE, AND RICHARD M. SCHIEKEN \\ The Childrens Medical Center, Division of Pediatric Cardiology, and Department of Human Genetics, The \\ Medical College of Virginia, Virginia Commonwealth University, Richmond, Virginia 23298
}

\begin{abstract}
We investigated the relative contributions of genetic, individual environmental, and shared environmental effects on 2,3-diphosphoglycerate (DPG) regulation in preadolescent children. In a population of 165 early pubescent boy and girl twin pairs (11.4 y old), of whom 63 were passive smokers, we asked: 1 ) Are there differences in the control of DPG levels between early pubertal boys and girls? 2) If present, are these differences influenced by exposure to passive cigarette smoke? Non-passive-smoking boys and girls had similar DPG levels. With exposure to passive smoke, DPG levels increased in boys $(p=0.02)$ but not in girls. Analysis of variance on DPG demonstrated a parental smoking effect $(p=0.008)$ and suggested an interactive effect between parental smoking and sex of the child $(p=0.08)$. Univariate genetic analyses suggested that genes operated at different magnitudes in boys $(9 \%)$ and girls $(39 \%)$ in explaining a significant portion of the variance in DPG. The magnitude of shared environmental influences was greater in boys (62\%) than in girls $(34 \%)$, whereas individual environmental effects were similar in boys $(29 \%)$ and girls $(26 \%)$. Early pubertal boys differ from girls in their regulation of DPG. Environmental stressors such as passive cigarette smoke may elicit different responses in males and females, even at an early age. The use of path analysis may provide important insights into the mechanisms and interactions of genetic and environmental effects that underly the childhood antecedents of atherosclerotic heart disease. (Pediatr Res 33: 645-648, 1993)
\end{abstract}

\section{Abbreviations}

DPG, 2,3-diphosphoglycerate

Systemic oxygen transport is accomplished using many integrated, self-regulated organ systems. Essential components of this system include pulmonary gas exchange, blood flow, Hb concentration, and $\mathrm{Hb}$ affinity for oxygen (1). Organic phosphates within the red cell decrease the oxygen affinity of $\mathrm{Hb}$. The most important quantitatively is DPG. The mechanism of the DPG effect on oxygen affinity involves binding of DPG to the two beta chains in the central cavity of deoxy $\mathrm{Hb}$, forcing a conformational shift that drives oxygen off. Oxyhemoglobin (as well as

Received September 3, 1992; accepted January 22, 1993.

Correspondence and reprint requests: William B. Moskowitz, M.D., Division of Pediatric Cardiology, Department of Pediatrics, The Children's Medical Center, Medical College of Virginia, P. O. Box 543, MCV Station, Richmond, VA 23298.

Supported by the National Institutes of Health, National Heart, Lung, and Blood Institute (Grants R29 HL-38878 and RO1-31010). carboxyhemoglobin) binds DPG poorly because of a stereochemically smaller central cavity created by changes in the quarternary structure of $\mathrm{Hb}$ with oxygenation (2). An increase in red-cell DPG compensates for an impairment in oxygen supply to tissue and is proportional to the increase in deoxyhemoglobin concentration.

Considerable variation in DPG levels, Hb levels, and oxygen affinity occurs in the normal population. A negative correlation has been observed between Hb levels and the levels of DPG in normal people $(3,4)$. Measurement of red-cell DPG levels has been used as a "functional biopsy" of the adequacy of tissue oxygenation (5). Whereas the correlation between DPG and $\mathrm{Hb}$ level may not be causal, feedback mechanisms appear to regulate them in tandem. Therefore, DPG may function as a monitor for environmental influences on systemic oxygen transport. Despite the importance of this organic phosphate, the inheritance of DPG levels in humans has not been studied in detail.

The purpose of the present study was to quantitate the relative contributions in children of both sexes of genetic and environmental effects (including passive cigarette smoking) to the variance of oxygen transport regulation. Specifically, we asked: 1) whether there were differences in the control of DPG levels between early pubertal boys and girls, and 2) if so, whether these differences were influenced by exposure to passive cigarette smoke. We hypothesized that sex differences in oxygen transport regulation exist at an early age and that males demonstrate a greater susceptibility to environmental influences.

\section{MATERIALS AND METHODS}

Population. As part of an ongoing longitudinal study of cardiovascular risk factors during adolescence, we recruited families with twins from nearby school systems. Eleven- and 121/2-y-old twins were ascertained from more than 75 middle schools of central Virginia within a 150 -mile radius. Information packets were mailed to the schools for distribution to parents of twins to maintain confidentiality from the investigators. The parents who replied by mail $(50 \%)$ were invited to participate. From a total population of 341 twin pairs, 165 twin pairs made up the sample for this study based on availability of DPG measurements. The sample population subjects were similar to those not included in weight, height, Tanner stage, and cigarette smoke exposure.

The families participated in a protocol that included the collection of data on family health and smoking histories and collection of blood samples for biochemical assays. The number of cigarettes smoked each day by the parents was recorded. Serum cotinine level was used both as the measure of smoke exposure and to verify non-passive-smoking status. No attempt was made to prescreen enrollees for the presence or absence of cardiovascular risk factors. Informed written consent, which had been approved by the Committee on the Conduct of Human Research 
of the Virginia Commonwealth University, was obtained from each family before it entered the study.

Procedures. Zygosity. Zygosity was assessed initially by questionnaire and confirmed by blood group and enzyme testing of all twins and their parents. HLA typing was also performed. With this battery of polymorphisms, the probability of dizygosity for concordant pairs typically is $<0.001(6)$.

Anthropometrics. Height and weight of each subject in stocking feet were measured with a stadiometer and digital scale, respectively. Sexual staging was performed using a five-scale score based upon Tanner's criteria (7).

Blood Samples. Whole blood was obtained by venipuncture, and red-cell DPG level was determined by standard colorimetric methods (8). The error variance for the measurement of DPG was $5 \%\left(R^{2}=0.95\right)$.

Statistical Analysis. Data are presented as mean \pm SD. Statistical differences between group means were assessed by pooled $t$ test. DPG values were found to be normally distributed using the Shapiro-Wilk statistic. For analysis, the subjects were divided into five groups by zygosity and sex. The designation twin 1 or twin 2 was by birth order with the exception of opposite-sex twin-pair groups, in which the male member was consistently designated as twin 1 . The methods of path analysis were used to specify the expected contributions of genetic and environmental variables to the variances and covariances of the five groups of twins $(9,10)$. We have previously described the use of univariate path analysis to quantitate the relative contributions of genes and environment to the variance of coronary risk factors in children $(11,13)$. This approach provides estimates and tests of significance for the parameters and provides a test of the assumptions implied in the model $(14,15)$. Path models were specified and fitted using the LISREL VII program for the structural analysis of covariance matrices $(16,17)$.

\section{RESULTS}

Age and anthropometric data are shown in Table 1. No differences were found between the sexes for age, weight, or height. Girls were more sexually mature than boys by Tanner staging.

Mean DPG levels for boys and girls, cross-classified with passive smoking status, are shown in Table 2. In 63 families, one or both parents were cigarette smokers at the time of evaluation. The total number of cigarettes smoked per day by the parents was similar for female and male twin pairs $(9.0 \pm 14.2$ versus $12.4 \pm 18.2$ cigarettes/d, $p=0.22$ ). None of the twins had ever smoked cigarettes.

Non-passive-smoking boys and girls had similar DPG levels. Overall, passive-smoking children demonstrated higher DPG levels than non-passive-smoking children. However, when analyzed by sex, the noted difference in passive-smoking children

Table 1. Age and anthropometric data

\begin{tabular}{lrcc}
\hline & \multicolumn{1}{c}{ Female } & Male & $p$ \\
\hline Age $(\mathrm{y})$ & $11.4 \pm 0.5$ & $11.4 \pm 0.5$ & NS \\
Wt $(\mathrm{kg})$ & $41.0 \pm 9.6$ & $41.1 \pm 10.3$ & NS \\
Ht $(\mathrm{cm})$ & $149.1 \pm 7.5$ & $148.9 \pm 8.0$ & NS \\
Tanner stage & $2.9 \pm 1.2$ & $2.4 \pm 1.1$ & 0.0001 \\
\hline
\end{tabular}

Table 2. Mean levels of DPG ( $\mathrm{mmol} / \mathrm{mL})$ by sex and passive smoking status*

\begin{tabular}{lllc}
\hline & \multicolumn{1}{c}{ Non-PS } & \multicolumn{1}{c}{ PS } & $p$ \\
\hline Both sexes & $2.01 \pm 0.28(102)$ & $2.12 \pm 0.27(63)$ & 0.01 \\
Males & $2.01 \pm 0.28(62)$ & $2.15 \pm 0.27(38)$ & 0.02 \\
Females & $2.01 \pm 0.26(40) \dagger$ & $2.08 \pm 0.28(25) \dagger$ & NS \\
\hline
\end{tabular}

* PS, passive smoking.

$\dagger$ Male-female mean difference was not significant $(p>0.05)$. Analyses are based on one twin per family. was accounted for by a significant elevation of DPG levels only in the boys. Analysis of variance on DPG demonstrated that there was an effect of the parents' smoking status $(p=0.008)$ and a suggestion of an interaction effect between parents' smoking and sex of the child $(p=0.08)$. Multiple linear regression analysis on DPG was performed to evaluate the fraction of the individual variation that was attributable to biologic traits that included: time since last meal, time since exercise, number of workouts per week, height, weight, age, and Tanner stage. None of these variables were found to be significant predictors of DPG levels.

Intraclass correlation coefficients for twin DPG, cross-categorized by sex and zygosity, are shown in Table 3. If family aggregation were totally due to genetic effects, dizygotic twin correlations would be expected to be one-half of monozygotic twin correlations. Conversely, if family aggregation were totally due to environmental effects shared by both twins, dizygotic twin correlations would be identical to monozygotic twin correlations. The data categorized by sex yielded distinct patterns. Femalefemale intrapair correlations were higher in monozygotic than in dizygotic twins ( $r=0.76$ versus 0.32 ), consistent with an important genetic effect. Male-male monozygotic and dizygotic twin-pair correlations were similar ( $r=0.61$ versus 0.73 ), consistent with an important shared environmental effect with little if any genetic influence. As expected, the opposite-sex dizygotic twin-pair correlation fell between those for same-sex dizygotic twin pairs.

Maximum-likelihood parameter estimates are presented for the full path model for male and female twins in Figure 1 . The regression of the phenotypes of the twins, DPG of twin 1 and twin 2 , on the latent variables [additive genes $(G)$, shared environment $(C)$, and individual environment $(E)]$ is specified by the path coefficients: $h$ (path from the genotype to DPG), c (path from the shared environment to DPG), and e (path from individual environment to DPG). The model allows for two kinds of sex differences in genetic and environmental effects. The relative magnitudes of the effects may differ between sexes, represented in the model by using $\mathrm{h}, \mathrm{c}$, and e for path coefficients in boys and $\mathrm{h}^{\prime}, \mathrm{c}^{\prime}$, and $\mathrm{e}^{\prime}$ for the corresponding values in girls. In addition, we allowed for the possibility that different genes and quantitatively different shared environments may contribute in each sex by allowing the correlation between genetic influences on males and on females $\left(r_{\mathrm{h}}\right)$ to be less than unity. Similarly, the correlation between shared environmental influences on males and females $\left(r_{c}\right)$ is allowed to be less than unity.

In the full model for female twins, the largest path coefficients were those associated with additive genetic effects and shared environment. Individual environmental effects were also significant. For male twins, the largest path coefficients are seen for shared environment, followed by individual environment with a small genetic effect.

The model-fitting results expressed as estimated percentages of the total phenotypic or DPG variance are presented in Table 4. The main sources of DPG variance are: genes $\left(h^{2}\right)$, shared environment $\left(\mathrm{c}^{2}\right)$, and individual environment $\left(\mathrm{e}^{2}\right)$. The $\chi^{2}$ goodness-of-fit statistic tests the agreement between observed and predicted statistics. A large $\chi^{2}$ indicates poor fit. The full model

Table 3. Twin-pair intraclass correlation coefficients for DPG

\begin{tabular}{lcc}
\hline & $\begin{array}{c}\text { No. of } \\
\text { pairs }\end{array}$ & DPG1-DPG2* \\
\hline Monozygotic twins & & \\
Male-male & 51 & 0.61 \\
Female-female & 45 & 0.76 \\
Dizygotic twins & & \\
$\quad$ Male-male & 13 & 0.73 \\
Female-female & 20 & 0.32 \\
Male-female & 36 & 0.65 \\
\hline
\end{tabular}

* DPG1-DPG2, across-twin correlation for DPG. 


\section{Full Model}

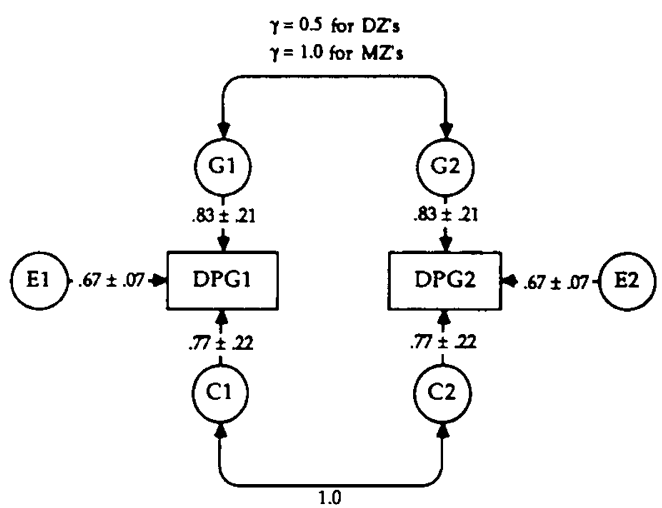

Twin 1
Twin 2

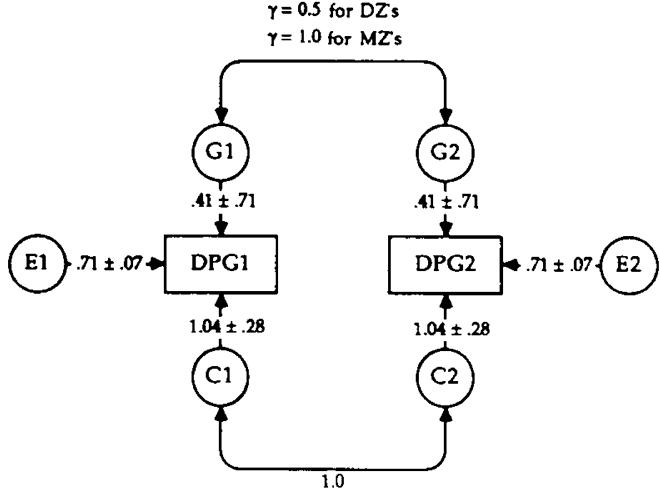

Twin 1
$\underline{\operatorname{Tw} 2} 2$

Females

Males

Fig. 1. Full path model for male and female twins. The phenotypes of twin 1 and twin $2(D P G 1, D P G 2)$ are modeled as being determined by additive genetic effects $(G 1, G 2)$, environmental effects shared by or common to both twins $(C 1, C 2)$, and environmental effects unique to each twin $(E 1, E 2)$. Maximum-likelihood parameter estimates for path coefficients $( \pm \mathrm{SEM})$ are presented for $h$ (path from genotype to DPG), $c$ (path from common environment to DPG), and $e$ (path from individual environment to DPG). $\gamma$, the correlation of genetic effects on twin 1 with those of twin $2 ; D Z$, dizygotic pairs; $M Z$, monozygotic pairs.

Table 4. Univariate analysis (partitioning of $D P G$ variance*)

\begin{tabular}{|c|c|c|c|}
\hline & $\begin{array}{c}\text { Sources of DPG } \\
\text { variance }\end{array}$ & Females $(\%)$ & Males $(\%)$ \\
\hline \multicolumn{4}{|c|}{ Full model $\left[\mathrm{hh}^{\prime} \mathrm{cc}^{\prime} \mathrm{ee}^{\prime} r_{\mathrm{h}}=0.5\right.$ (fixed)] } \\
\hline$\chi^{2}=8.14$ & $h^{2}$ & $39.4 \pm 20.0$ & $9.6 \pm 32.7$ \\
\hline$d f=9$ & $c^{2}$ & $34.5 \pm 19.6$ & $61.7 \pm 33.1$ \\
\hline$p>0.50$ & $\mathrm{e}^{2}$ & $26.1 \pm 5.5$ & $28.7 \pm 5.8$ \\
\hline \multicolumn{4}{|c|}{ Best-fitting model $\left(\mathrm{cc}^{\prime} \mathrm{ee}^{\prime}\right)$} \\
\hline$\chi^{2}=12.44$ & $h^{2}$ & Not in model & Not in model \\
\hline$\hat{d f}=11$ & $c^{2}$ & $62.8 \pm 12.6$ & $65.4 \pm 12.9$ \\
\hline$p>0.33$ & $\mathrm{e}^{2}$ & $37.1 \pm 6.2$ & $34.6 \pm 5.9$ \\
\hline
\end{tabular}

* Percentage of variance estimates are \pm SEM; $h^{2}$, genes; $c^{2}$, shared environment; and $\mathrm{e}^{2}$, individual environment.

suggests a greater genetic effect in the girls. A significant shared environmental effect predominates in the boys. Individual environmental effects are significant and similar in both sexes. The best-fitting four-parameter model ( $\left.\mathrm{cc}^{\prime} \mathrm{ee}^{\prime}\right)$ excluded genetic effects. This model indicates that the same types of effects (shared environment and individual environment) are working in both boys and girls and that the magnitudes of the effects are comparable. The best-fitting five-parameter model $\left(\mathrm{h}^{\prime} \mathrm{cc}^{\prime} \mathrm{ee}^{\prime}\right)$ would suggest that genetic effects are active in the girls but not in the boys at this age, an interpretation consistent with the pattern of observed correlations. However, this model did not fit significantly better than the best-fitting four-parameter model $\left(\chi^{2}\right.$ difference: $3.39,1 d f ; p=0.066$ ). We suspect that a larger sample of like-sexed dizygotic females may provide evidence for a significant genetic effect within females.

\section{DISCUSSION}

We found that early pubertal boys differ from girls in their regulation of DPG. Without cigarette-smoke exposure, boys and girls have similar DPG levels. Chronic cigarette-smoke exposure elevates DPG levels in boys but not girls.

Sex differences in oxygen delivery are known to exist in adults, with lower $\mathrm{Hb}$ levels, lower oxygen affinity, and higher DPG levels in females (18). The lower blood-oxygen affinity and redcell mass in females is associated with a higher DPG level as well as a younger red blood cell population (19). Higher red-cell glucose consumption, possibly due to greater hexokinase activity, may account for the higher adult female DPG levels (20). Our finding of similar DPG levels in early pubertal boys and girls who are not exposed to cigarette smoke suggests that gene actions, which determine the activity of various erythrocyte glycolytic enzymes, may change during puberty. In another study (21), DPG was found to be significantly higher in sexually mature females than in males of similar age, and no sex differences existed before puberty. These data also suggest a sex hormoneand maturation-induced influence in the development of the red-cell oxygen transport system. The comparison of DPG levels in younger prepubertal and older, mature, non-smoke-exposed boys and girls may provide further insight into the mechanisms of oxygen transport regulation.

Chronic passive cigarette-smoke exposure was associated with an elevation in DPG levels in boys but not girls. We have previously shown (22) that in families with adult smokers, the DPG level in the children correlated directly with the serum thiocyanate level and the total number of cigarettes smoked by the parents. DPG levels in preadolescent boys increased in a dose-dependent relationship with the amount of passive cigarette-smoke exposure. Their serum thiocyanate and cotinine levels correlated with the total number of cigarettes smoked each day by their parents. Passive-smoking boys and girls had similar elevations of serum thiocyanate. The intratwin pair correlation for thiocyanate was also high $(r=0.94, p<0.0001)$. This demonstrates that twins from a smoking family have similar exposures to home environmental cigarette smoke.

In hypoxic states, red blood cell DPG levels increase to meet tissue oxygen requirements. Hypoxia has been implicated as the triggering mechanism for DPG synthesis in active cigarette smokers $(23,24)$. A hypoxia-driven mechanism triggering DPG synthesis is likely in passive-smoking individuals. Although the boys and girls in the present study had similar amounts of passive cigarette-smoke exposure, the discrepant DPG responses suggest sex-specific patterns of reactivity of the systemic oxygen transport system. Boys demonstrated a greater reactivity to this environmental stressor. This may have occurred because environmental factors predominated over genetic factors in the adjustment of DPG levels in boys.

DPG represents an effective, environmentally influenced genetic regulator of systemic oxygen transport. Using the power of path analysis, we have attempted to quantitate the genetic contribution and partition the relative contributions of individual 
(including laboratory measurement variation) and shared environmental effects on DPG regulation. Despite this large sample population, which included opposite-sex twin pairs, our observations are preliminary. A larger sample will be needed to confirm these findings. The suggested sex differences in the regulation of DPG may be a consequence of the more advanced sexual maturation of the girls at this age. The extent to which these differences persist or whether genetic control will begin to emerge in males with maturity is being examined in ongoing longitudinal studies.

Cigarette smoking is a powerful independent risk factor associated with premature coronary heart disease in men, with the greatest risk occurring in younger age groups $(25,26)$. We have previously shown (22) that passive-smoking preadolescent boys had lower levels of $\mathrm{HDL}_{2}$ cholesterol, which were related to the number of cigarettes smoked daily by the parents of the boys. The lowest $\mathrm{HDL}_{2}$ cholesterol levels were found in boys exposed to the highest number of cigarettes smoked by their mothers. The $\mathrm{HDL}_{3}$ subfraction was similarly lower in the passive-smoking children with greater differences seen in the girls. Both HDL subfractions have strong inverse and independent associations with myocardial infarction $(13,27)$. As a consequence of their lowered HDL cholesterol subfractions, children with long-term exposure to passive smoke may be at elevated risk for the development of premature coronary heart disease.

This study estimates the partitioning of the variance in oxygen transport regulation into genetic and environmental influences by use of univariate genetic path analyses. A larger sample of twins will provide more confidence in the tests for sex differences in the genetic or environmental effects on DPG regulation. Environmental factors appear to predominate over genetic factors in the adjustment of DPG levels in boys. Environmental factors other than passive smoke exposure are likely to impact on DPG control. Other lines of investigation, such as gene by environment interaction (i.e. genetic background influence on the response to a given environmental stimulus), may provide some answers. Multivariate genetic path analyses that include sexual maturity as well as cholesterol subfractions may have the potential to explain the genetic and environmental interrelationships of these variables. The use of path analysis may provide important insights into the mechanisms and interactions of genetic and environmental effects that underly the childhood antecedents of atherosclerotic heart disease.

Acknowledgments. The authors thank A. Cook, L. Stevenson, B. Toms, A. Frayser, C. Darlington, C. Martin, L. Davidson, and W. Smith for their technical expertise.

\section{REFERENCES}

1. Finch CA, Lenfant C 1976 Oxygen transport in man. N Engl J Med 286:407415

2. Benesch R, Benesch RE 1969 Intracellular organic phosphates as regulators of oxygen release by hemoglobin. Nature 221:618-622

3. Eaton JW, Brewer GJ 1968 The relationship between red cell 2,3-diphospho- glycerate and levels of hemoglobin in the human. Proc Natl Acad Sci USA $61: 756-760$

4. Hjelm M 1969 The content of 2,3-diphosphoglycerate and some other phosphocompounds in human erythrocytes from healthy adults and subjects with different kinds of anemia. Forsvarsmedecin 5:219-225

5. Valeri CR, Fortier NL 1969 Red-cell 2,3-diphosphoglycerate and creatine levels in patients with red-cell mass deficits or with cardiopulmonary insufficiency. N Engl J Med 281:1452-1455

6. Miller JZ, Norton JA, Nance WE 1977 Vitamin C and growth. JAMA 238:937938

7. Tanner JM 1962 Growth at Adolescence, 2nd Ed. Blackwell Scientific, Oxford, $\mathrm{UK}$

8. Rose Z 1970 Enzymes controlling 2,3-diphosphoglycerate in human erythrocytes. Fed Proc 29:1105-1111

9. Morton NE 1974 Analysis of family resemblance. I. Introduction. Am J Hum Genet 26:318-330

10. Rice J, Cloninger RC, Reich T 1978 Multifactorial inheritance with cultural transmission and assortative mating. I. Description and basic properties of unitary models. Am J Hum Genet 30:618-643

11. Schieken RM, Eaves LJ, Hewitt JK, Mosteller M, Bodurtha JN, Moskowitz WB, Nance WE 1989 Univariate genetic analysis of blood pressure in children (The Medical College of Virginia Twin Study). Am J Cardiol 64:1333-1337

12. Bodurtha JN, Mosteller M, Hewitt JK, Nance WE, Eaves LJ, Katz S, Schieken RM 1990 Genetic analysis of anthropometric measures in 11-year-old twins. Pediatr Res 28:1-4

13. Bodurtha JN, Chen CW, Mosteller M, Nance WE, Schieken RM 1991 Genetic and environmental contributions to cholesterol and its subfractions in 11year old twins: The Medical College of Virginia Twin Study. Arteriosclerosis Thrombosis 11:844-850

14. Eaves LJ, Last K, Young PA, Martin NG 1978 Model fitting approaches to the analysis of human behavior. Heredity $41: 249-320$

15. Rao DC, Morton NE, Yee S 1974 Analysis of family resemblance. II. A linear model for family correlation. Am J Hum Genet 26:331-359

16. Joreskog KG, Sorbom D 1989 Lisrel VII: A Guide to the Program and Applications, 2nd Ed. SPSS Inc, Chicago

17. Martin NG, Eaves LJ, Jardine R, Heath AC, Feingold LF, Eysenck J 1986 Transmission of social attitudes. Proc Natl Acad Sci USA 83:4364-4368

18. Brewer GJ 1974 2,3-DPG and erythrocyte oxygen affinity. Annu Rev Med 25:29-38

19. Samaja M, Rovida E, Motterlini R, Tarantola M 1991 The relationship between the blood oxygen transport and the human red cell aging process. Adv Exp Med Biol 307:115-23

20. Brewer GJ, Eaton JW, Weil JV, Grover RF 1970 Red cell metabolism and function. Adv Exp Med Biol 6:95-114

21. Humpeler E, Vogel S, Schobersberger W, Mairbaurl H 1989 Red cell oxygen transport in man in relation to gender and age. Mech Ageing Dev 47:22939

22. Moskowitz WB, Mosteller M, Schieken RM, Bossano R, Hewitt JK, Bodurtha JN, Segrest JP 1990 Lipoprotein and oxygen transport alterations in passive smoking preadolescent children. Circulation 81:586-592

23. Rao GL, Rao AK, Pattabiraman TN 1979 Studies on 2,3-diphospho-glycerate levels in blood. Indian J Med Res 70:483-486

24. Dinman BD, Eaton JW, Brewer GJ 1974 Effects of carbon monoxide on 2,3 diphosphoglycerate concentrations in the erythrocyte. Ann NY Acad Sci USA 174:246-250

25. Grundy SM, Greenland P, Herd A, Huebsch JA, Jones RJ 1987 Cardiovascular and risk factor evaluation of healthy American adults. Circulation 75:1339A$1362 \mathrm{~A}$

26. Kannel WB 1981 Update on the role of cigarette smoking in coronary artery disease. Am Heart J 101:319-328

27. Buring JE, O'Connor GT, Goldhaber SZ, Rosner B, Herbert PN, Blum CB Breslow JL, Hennekens CH 1992 Decreased HDL2 and HDL 3 cholesterol, Apo A-I and Apo A-II, and increased risk of myocardial infarction. Circulation 85:22-29

28. Bouchard C, Tremblay A, Despres JP, Nadeau A, Lupien PJ, Theriault G, Dussault J, Moorjani S, Pinault S, Fournier G 1990 The response to longterm overfeeding in identical twins. N Engl J Med 322:1477-1482 